\title{
Surface effects on nitrogen vacancy centers neutralization in diamond
}

\author{
Arthur N. Newell, ${ }^{1,2}$ Dontray A. Dowdell, ${ }^{1,2}$ and D. H. Santamore ${ }^{1,2, a)}$ \\ ${ }^{1)}$ Department of Physics and Engineering Physics, Delaware State University, Dover, Delaware 19901, \\ $U S A$ \\ ${ }^{2)}$ ITAMP, Harvard-Smithsonian Center for Astrophysics, Cambridge, Massachusetts 02138, \\ $U S A$
}

(Dated: 16 June 2021)

The performance of nitrogen vacancy $\left(\mathrm{NV}^{-}\right)$based magnetic sensors strongly depends on the stability of nitrogen vacancy centers near the diamond surface. The sensitivity of magnetic field detection is diminished as the $\mathrm{NV}^{-}$turns into the neutralized charge state $\mathrm{NV}^{0}$. We investigate the neutralization of $\mathrm{NV}^{-}$and calculate the ratio of $\mathrm{NV}^{0}$ to total $\mathrm{NV}\left(\mathrm{NV}^{-}+\mathrm{NV}^{0}\right)$ caused by a hydrogen terminated diamond with a surface water layer. We find that $\mathrm{NV}^{-}$neutralization exhibits two distinct regions: near the surface, where the $\mathrm{NV}^{-}$ is completely neutralized, and in the bulk, where the neutralization ratio is inversely proportional to depth following the electrostatic force law. In addition, small changes in concentration can lead to large differences in neutralization behavior. This phenomenon allows one to carefully control the concentration to decrease the $\mathrm{NV}^{-}$neutralization. The presence of nitrogen dopant greatly reduces $\mathrm{NV}^{-}$neutralization as the nitrogen ionizes in preference to $\mathrm{NV}^{-}$neutralization at the same depth. The water layer pH also affects neutralization. If the $\mathrm{pH}$ is very low due to cleaning agent residue, then we see a change in the band bending and the reduction of the 2-dimensional hole gas (DHG) region. Finally, we find that dissolved carbon dioxide resulting from direct contact with the atmosphere at room temperature hardly affects the $\mathrm{NV}^{-}$neutralization.

\section{INTRODUCTION}

Nitrogen vacancy $\left(\mathrm{NV}^{-}\right)$center diamonds have recently become the subject of intense research for emergent quantum technologies such as metrology, including nanoscale sensing ${ }^{1-11}$; magnetometry ${ }^{12-16}$; and singlespin $\mathrm{NMR}^{16,17}$. Since $\mathrm{NV}^{-}$spin is extremely sensitive to changes in the magnetic field, $\mathrm{NV}^{-}$center diamonds can be used to detect paramagnetic objects optically and nondestructively. Ideally, it is even possible to detect a single spin. $\mathrm{NV}^{-}$-based devices can be operated at room temperature and in air, which is a great advantage for biological applications, because specimens will not be destroyed by the scanning process. In recent years, considerable efforts have been made to use $\mathrm{NV}^{-}$center diamonds for ultra-sensitive nanoscale magnetic sensor heads $2,4,18$ and sensor arrays $8,19,20$. These sensors take advantage of the long-lived spin state of single NV centers to detect small magnetic fields ${ }^{4}, 5$. Many nanoscale imaging experiments using an NV center have provided magnetic images of various objects, including disk drive media ${ }^{21,22}$, magnetic vortices ${ }^{23}$, a single electron $\operatorname{spin}^{24}$ and magnetotactic bacteria ${ }^{20}$. It should be emphasized that magnetic sensors rely on an NV center having an extra electron $\left(\mathrm{NV}^{-}\right)$. The neutralized form, $\mathrm{NV}^{0}$, is not useful as a magnetic field sensor.

To detect a magnetic field in a sample, either the sample is moved over the $\mathrm{NV}^{-}$center embedded in the bulk diamond, or an $\mathrm{NV}^{-}$embedded cantilever tip scans over the sample, depending on whether the $\mathrm{NV}^{-}$center is implanted at a shallow depth in the substrate or the $\mathrm{NV}^{-}$ center is located close to the apex of a tip. In both cases,

a) Electronic mail: dsantamore@desu.edu. it is very important to maintain a sufficiently strong dipole-dipole coupling between the $\mathrm{NV}^{-}$center and the target spin for detection. The distance between the $\mathrm{NV}^{-}$ center and the sample surface must be $10 \mathrm{~nm}$ or less, and ideally less than $5 \mathrm{~nm}$. However, shallow $\mathrm{NV}^{-}$centers $(<5 \mathrm{~nm})$ tend to become neutralized to $\mathrm{NV}^{0}$, rendering magnetic field detection impossible ${ }^{25,26}$. The hydrogen surface termination commonly used for semiconductor processing bends the band diagram and creates a two dimensional hole gas (2-DHG) near the surface, which accelerates the neutralization of $\mathrm{NV}^{-}$centers $^{27-30}$. Other terminations, such as oxygen and fluorine, do not have the energy band bending problem. However, oxygen termination has its own problem. How oxygen bonds with carbon affects the band diagram drastically. If a $\mathrm{C}-\mathrm{OH}$ bond is formed, the surface interface has the same problem with the hydrogen termination case causing the band bending and accelerating the neutralization of $\mathrm{NV}^{-}$centers. On the other hand, fluorine termination is reported to encounter severe blinking or bleaching ${ }^{25,31}$. The detailed analyses about different surface terminations and the bond structures are discussed by Kaviani et al.32. In addition, NV centers interacting with paramagnetic contaminants such as water molecules on the surface can decrease the sensitivity of a magnetometer ${ }^{16,33-35}$. While experimental progress in achieving ultra-high precision devices has been quite impressive, the theory of bulk impurity interactions with NV centers and surface effects is not well understood.

In this paper, we investigate the surface effects of $\mathrm{NV}^{-}$ neutralization in diamond. Sec. II introduces the physical model (an $\mathrm{NV}^{-}$embedded diamond with a hydrogen terminated surface and a water layer) and equations to be solved (the Poisson equation, the Schrödinger-Poisson equation, and the Poisson-Boltzmann equation), and describes the numerical scheme to evaluate our model. Sec. 
III shows our results and discusses the physical implications. We examine the effects on $\mathrm{NV}^{-}$neutralization with depth, concentration, addition of nitrogen, and $\mathrm{pH}$ of the water layer. Finally, Sec. IIIG concludes our discussions.

\section{METHODS}

\section{A. Model Structure}

$\mathrm{NV}^{-} \mathrm{s}$ are created by doping nitrogen in diamond through either chemical vapor deposition or ion implantation. A nitrogen substitutes for a carbon atom in a diamond unit cell. Then a vacancy next to the nitrogen in the unit cell is formed by thermal annealing and an $\mathrm{NV}^{-}$is formed ${ }^{36-38}$. The typical yield of $\mathrm{NV}^{-}$from nitrogen dopant is $0.1 \%-1 \%$; however, there are some recent report of $10 \%$ and even $50 \% \frac{39}{3}$ at the time of writing.

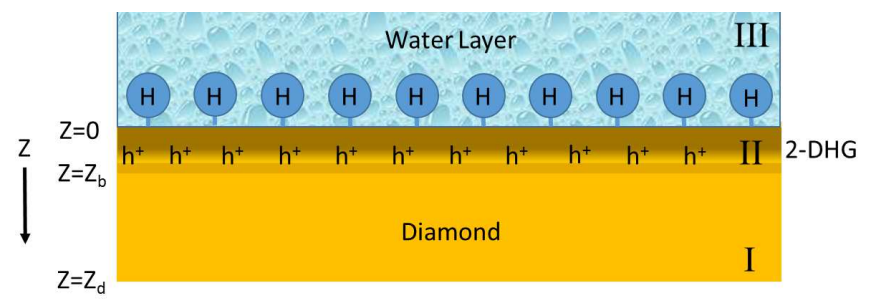

FIG. 1. Schematic representation of our model. The model consists of a bulk diamond region (I), the hole $\left(\mathrm{h}^{+}\right)$accumulated region near the surface (II), and the surface of the diamond with hydrogen termination and a water layer (III).

Figure 1 shows a schematic of our model. We study a hydrogen-terminated (100) diamond surface containing an $\mathrm{NV}^{-}$center at room temperature and one atmospheric pressure as these are the typical conditions at which most magnetic sensing $\mathrm{NV}^{-}$center diamond devices operate. A thin water layer (usually a mono layer) condenses on the surface from the water vapor in surrounding air. In addition, carbon dioxide in the air may be dissolved in the water layer, changing the $\mathrm{pH}$ of the water. The effect of $\mathrm{pH}$ changes on $\mathrm{NV}^{-}$neutralization will be described in Sec. IIIF.

The negative electron affinity (NEA) of hydrogenterminated surface in vacuum is reported to be $\chi=-1.3$ $\mathrm{eV}^{\underline{40}}$.41. The NEA leads to the depletion of electrons and the creation of a hole accumulation layer (2-DHG), represented by $\left(\mathrm{h}^{+}\right)$in Fig. 1. The depletion of electrons continues until the Fermi energy of the $\mathrm{NV}^{-}$center diamond levels with the chemical potential of the atmosphere plus the water layer. This causes the energy bands to bend. With the presence of a surface water layer, the NEA can be even lower, further enhancing the bending.

There are three distinct regions that require different approaches to analyze $\mathrm{NV}^{-}$neutralization (see Fig. 1):
Region I is the bulk diamond away from the surface and the 2-DHG, Region II is the depletion region including the 2-DHG, and Region III is the hydrogen terminated surface with the water layer. In Fig. 1 $z$ is the depth from the surface. Two interfaces separating two regions are $z=0$ (the diamond and the surface) $z=z_{b}$ (the depletion region and bulk diamond), and $z=z_{d}$ is the edge of the diamond. We begin our analysis with Region II for mathematical convenience as well as it being the most important region where the hole accumulation and band bending occurs.

Region II is where the holes accumulate in the range $0<z<z_{b}$. The depletion region has two forces acting on it: the electrostatic force and the exchange and correlation force $\underline{42}$. The electrostatic force is due to the charge density of other conduction electrons, the ionized donors, and the dipole interaction with the surface water layer and contaminants dissolved in water. The exchange and correlation forces result from other conduction electrons, and between a conduction electron and the valence electrons. The exchange and correlation forces are usually small; however, they become important for analyzing band bending, the charge profile, the density of states, and the subband structure. To account for these forces, we use the Schrödinger-Poisson equation. The Schrödinger equation is used for the states of the conduction holes, providing the charge density in terms of the occupied states and the uniform-background charge density, while Poisson's equation gives the potential in terms of the charge density calculated by the Schrödinger equation ${ }^{42}$.

The Schrödinger equation for the mobile states is

$$
\left[-\frac{\hbar^{2}}{2 m_{n}} \frac{d^{2}}{d z^{2}}+V(z)\right] \psi_{i}(z)=\varepsilon_{i} \psi_{i}(z)
$$

where $z$ denotes the depth from the surface, $\psi_{i}(z)$ is the hole sub-band $i$ state wave function with the eigenenergy $\varepsilon_{i}$ and

$$
V(z)=q \Phi(z)+V_{h}
$$

Here $q$ is the charge, $\Phi(z)$ is the electrostatic potential, $V_{h}$ is the effective potential energy associated with the interface discontinuity barrier (the difference between the pinned valence band maximum at the interface and the Fermi energy) which is reported to be $1.68 \mathrm{eV}^{\underline{43}}$.

The boundary conditions are Dirichlet

$$
\psi_{i}(0)=\psi_{i}\left(z_{b}\right)=0,
$$

where $z=0$ is the interface. We set $z=z_{b}$ to be much deeper than the 2-DHG layer into the bulk diamond so that $z_{b}$ is well-away from the 2-DHG accumulation region, which allows us to contain the majority of the band bending, as well as allows the wavefunction to be extended beyond the depletion if needed. $m_{n}$ is the effective mass of holes, where $n$ runs over the heavy hole $(\mathrm{HH})$, light hole ( $\mathrm{LH})$ and split-off (SO) bands. Solutions 
of Eq. (11) are used to calculate the charge density in the Poisson equation

$$
\begin{aligned}
\frac{d^{2} \Phi}{d z} & =-\frac{\rho(z)}{\epsilon} \\
& =-\frac{1}{\epsilon} 2 q\left[\sum_{m, b} \sum_{i} f\left(\varepsilon_{i}\right) \psi_{i}^{*}(z) \psi_{i}(z)\right]+e N_{0} \\
& =\frac{2 q}{\epsilon} \sum_{i} N_{i} \psi_{i}^{2}(z)+e N_{0} .
\end{aligned}
$$

Here $\Phi$ is the electrostatic potential, $\rho(z)$ is the charge density, $\epsilon=\epsilon_{r} \epsilon_{0}=5.7 \epsilon_{0}$ is the permittivity of diamond ( $\epsilon_{0}$ is the vacuum permittivity and $\epsilon_{r}$ the relative permittivity.) The factor of two is the spin degeneracy, $f\left(\varepsilon_{i}\right)$ is the probability of the particle having energy $\varepsilon_{i}$ when the Fermi energy is at $E_{F}$, and $m$ and $b$ stand for mobile and bound states. The second term $e N_{0}$ is the uniform background charge density. The spatial density of particle $N_{i}$ is determined by the effective density of states and the Fermi-Dirac distribution. We assume the dielectric constant is position independent.

The boundary conditions for the Poisson equation are

$$
\begin{aligned}
\Phi\left(z_{b}\right) & =\Phi_{0}, \\
-\left.\frac{d \Phi}{d z}\right|_{z=0} & =E_{0},
\end{aligned}
$$

where $E_{0}$ is the electric field at the surface of the diamond. The Schrödinger-Poisson equation can be solved only numerically.

Now considering region III $(z<0)$, where electrolyte covers the surface, we need to consider mobile charges such as molecules and ions rather than the fixed ions of the solid. The Poisson equation is no longer applicable as molecules and ions freely move about within the solution at finite temperatures in thermal motion, even at equilibrium. The Poisson-Boltzmann equation describes the distribution of the electric potential in the solution in the direction normal to the charged surface.

Consider the situation where the interface $(z=0)$ has the surface charge density $\sigma$. If $\Phi(\mathbf{z})$ is the electrostatic potential, the charge density of the system is

$$
\rho(z)=\sigma \delta(z)+q n_{0} \exp \left[-\frac{q \Phi}{k_{B} T}\right],
$$

where $k_{B}$ is the Boltzmann constant, $T$ is the temperature, and $n_{0}$ is the ion concentration very far from the interface). The freedom of movement of ions in the electrolyte is accounted for by Boltzmann statistics. Substituting Eq. 7 into the Poisson equation gives the PoissonBoltzmann equation,

$$
\begin{aligned}
\frac{d^{2} \Phi}{d z^{2}} & =-\frac{1}{\epsilon}\left\{\sigma \delta(z)+q n_{0} \exp \left[-\frac{q \Phi}{k_{B} T}\right]\right\} \\
& \Rightarrow \frac{d^{2} \Phi}{d z^{2}}+\frac{q n_{0}}{\epsilon} \exp \left[-\frac{q \Phi}{k_{B} T}\right]=-\frac{\sigma}{\epsilon} \delta(z)
\end{aligned}
$$

We have assumed that (a) the energy in the Boltzmann statistics depends only on the electrostatic energy, (b) $\epsilon$ has no position dependence, (c) point-like ions interact via their mean field, and (d) the water layer is treated as a structureless continuum. With these assumptions, the Poisson-Boltzmann equation becomes the nonlinear Guoy-Chapman model for the electrolyte $\underline{4-46}$. Although structural properties of the liquid such as the sizes of ion and solvent and their interactions can influence the ion energy, they are negligible since their effects are small. In addition, we are interested in $\mathrm{NV}^{-}$neutralization and not the dynamics of the electrolyte itself. If we want to know the details of electrolyte distributions, then we would need to use molecular dynamics simulations to introduce liquid structure rather than solving a non-linear Gouy-Chapman model. However, molecular dynamics simulations are beyond the scope of this paper since we are more concerned with the physics of the diamond (Region I and II) than with the details of the electrolyte region (Regions III).

One of the boundary conditions is obtained by integrating Eq. 8 over an infinitesimal interval around $z=0$. We also impose the boundary condition that the electric field $E(z) \rightarrow 0$ as $z \rightarrow \infty$. If $q \Phi / k_{B} T \ll$ 1 , then we can Taylor expand $\exp \left[-q \Phi(\mathbf{r}) / k_{B} T\right]$ as $\exp \left[-q \Phi(\mathbf{r}) / k_{B} T\right] \simeq 1-q \Phi(\mathbf{r}) / k_{B} T$, which is the Debye-Hückel approximation. The Debye-Hückel approximation makes the Poisson-Boltzmann equation analytically solvable. However, in our case, the potential calculated from the numerical solution gives $q \Phi / k_{B} T>1$, so that the approximation does not apply. We must therefore solve the full equation numerically.

Finally, Region I contains the majority of the bulk diamond and is far from the surface and the 2-DHG. This region can be solved by a simple Poisson equation. The boundary conditions for the Poisson equation are

$$
\begin{gathered}
\Phi\left(z_{b}\right)=\Phi_{0}, \\
-\left.\frac{d \Phi}{d z}\right|_{z=z_{d}}=0 .
\end{gathered}
$$

\section{B. The role of a surface water layer}

So far, we have always incorporated a water layer as an integral part of the system in our model since water molecules are polar molecules. Macroscopically, the water layer will function as an electric dipole layer on the surface that will behave like the surface charge density $\sigma \underline{47}, \underline{48}$. As a very rough estimate of the surface charge density, let us model the monolayer of water as a dipole layer, two oppositely charged surfaces with area charge densities $\pm \sigma$. The dipole moment of a molecule is $\mathbf{p}=q_{\mathrm{eff}} \mathbf{b}$, where $b$ is the molecule's length and $q_{\mathrm{eff}}$ is the effective charge. Assuming that each molecule occupies an area $S$ of the interface, we can calculate a uniform electric dipole moment per unit area $\tau$, where $\tau=p / S$. 
In the macroscopic limit, $b \rightarrow 0$ and $\sigma \rightarrow \infty$ with $\tau=\sigma b$ held constant,

$$
\frac{p}{S}=\frac{q_{\mathrm{eff}} b}{S}=\sigma b
$$

which leads to $\sigma=q_{\text {eff }} / S$. The magnitude of the total dipole moment of a water molecule is $p=6.1 \times 10^{-30}$ $\mathrm{C} \cdot \mathrm{m}$, and the size is $0.275 \mathrm{~nm}$, the effective charge is $6.38 \times 10^{-20} \mathrm{C}$ and the uniform surface charge density is $8.4 \times 10^{-5} \mathrm{C} \cdot \mathrm{cm}^{-2}$, which is comparable to the effective surface charge that can cause the same band bending as the one caused by the hydrogen termination. Therefore, the water layer contributes to the band bending and affects the neutralization of $\mathrm{NV}^{-}$, and we need to include the surface water layer.

\section{Numerics}

We calculate the $\mathrm{NV}^{-}$neutralization numerically since the equations we have cannot be solved analytically. We are particularly interested in the effects on $\mathrm{NV}^{-}$neutralization of a hydrogen terminated surface with a water layer. We consider only nitrogen dopant and $\mathrm{NV}^{-}$and disregard other bulk contaminants, such as ${ }^{13} \mathrm{C}$ and $\mathrm{B}$, in order to isolate the neutralization caused by surface effects.

Diamond is an insulator and has an energy gap of $5.47 \mathrm{eV}$. We set the $\mathrm{N}^{0 /+}$ transition level to $1.7 \mathrm{eV}$ below the conduction band minimum (CBM) and the $\mathrm{NV}^{-/ 0}$ transition level to $2.67 \mathrm{eV}$ below the conduction band minimum ${ }^{49}$. Since many applications operate $\mathrm{NV}^{-}$ center diamond devices at room temperature, we take $T=298.15 \mathrm{~K}$.

The $\mathrm{pH}$ of the water layer is taken to be 7 to simulate a layer of pure water condensed on the surface. Realistically, the water layer can be slightly acidic ( $\mathrm{pH}$ of around 6) when $\mathrm{CO}_{2}$ is dissolved in the water when it is in contact with air. We discuss that such changes in $\mathrm{pH}$ produce negligible differences in the neutralization of $\mathrm{NV}^{-}$in Sec. IIIF

The surface of hydrogen terminated diamond is known to be hydrophobic. The diamond and water layer are generated within a 1-D model, as the neutralization of the $\mathrm{NV}^{-}$depends primarily on the depth of the $\mathrm{NV}^{-}$ from the surface.

As mentioned in Sec. IIA, our model uses the nonlinear Poisson equation in the bulk diamond (Region I), Schrödinger-Poisson equation in the depletion region (Region II), and the Poisson-Boltzman equation in the water layer (Region III). We use the software package 'nextnano ${ }^{3}$ ' for numerical evaluations ${ }^{50}$. Nextnano ${ }^{3}$ iteratively solves the Poisson equation self-consistently within the discretized diamond and water regions on a non-uniform grid by the finite-differences method. The Schrödinger-Poisson equation couples the Poisson equation to the Schrodinger equation through the charge density in Eqs. (1) and (4) within the diamond region. The
Poisson-Boltzmann equation is solved self-consistently using the same numerical scheme as in diamond.

\section{RESULTS}

\section{A. Band Diagram}

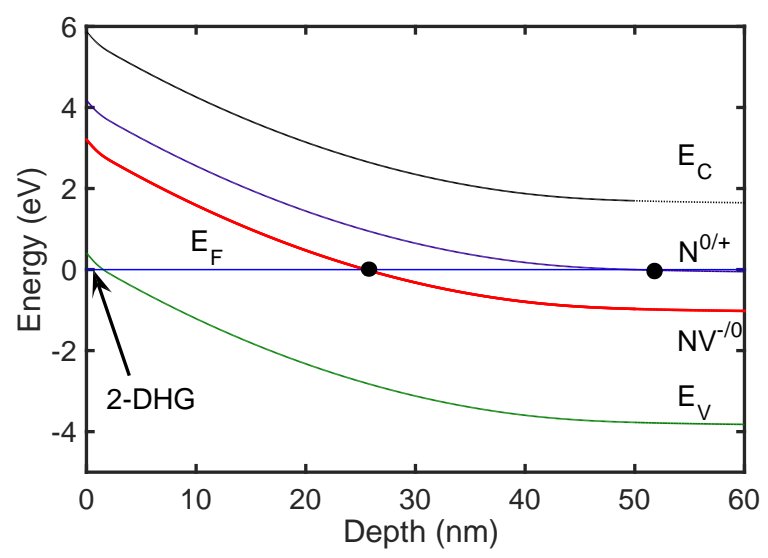

FIG. 2. Band diagram for diamond containing nitrogen and $\mathrm{NV}^{-}$. All energy levels calculated relative to a Fermi Level at $0 \mathrm{eV}$. The region where $\mathrm{E}_{V}$ crosses $\mathrm{E}_{F}$ is marked. This is the $2-\mathrm{DHG}$ region. The black dots represent the depths up to which nitrogen and $\mathrm{NV}^{-}$are completely neutralized.

Figure 2 shows the numerically evaluated band diagram for a diamond containing a homogeneous doping profile of both nitrogen and $\mathrm{NV}^{-}$. The diamond is 150 $\mathrm{nm}$ in depth and is doped with a nitrogen concentration of $10^{18} \mathrm{~cm}^{-3}$ and an $\mathrm{NV}^{-}$concentration of $10^{17} \mathrm{~cm}^{-3}$. These particular concentrations are used to simulate a $10 \%$ yield of $\mathrm{NV}^{-}$from nitrogen dopant. The typical yield of $\mathrm{NV}^{-}$is $0.1 \%-10 \%$. The Fermi level, $E_{F}$, is set to $0 \mathrm{eV}$ for convenience.

Hydrogen surface termination causes band-bending. A 2-DHG as a result of band-bending is shown in the figure. In Fig. 2, the 2-DHG is spread to the depth of around $1 \mathrm{~nm}$ from the surface. The points where the $\mathrm{N}^{0 /+}$ and $\mathrm{NV}^{-/ 0}$ transition lines cross the Fermi level are marked by black dots in Fig. 2] These points reflect $100 \%$ ionization of $\mathrm{N}^{0}$ to $\mathrm{N}^{+}$and $100 \%$ neutralization of $\mathrm{NV}^{-}$to $\mathrm{NV}^{0}$, respectively. The depletion region depth is much smaller than the complete ionization/neutralization points depths. However, the total charge of accumulated holes roughly equals that of ionized $\mathrm{N}$ plus neutralized $\mathrm{NV}^{-}$.

\section{B. Depth Dependence}

For an $\mathrm{NV}^{-}$-based device to function as a magnetic sensor, the $\mathrm{NV}^{-}$must be placed near the surface. The magnetic field sensitivity is directly related to the depth 
of an $\mathrm{NV}^{-}$. However, shallow $\mathrm{NV}^{-} \mathrm{s}$ tend to become neutralized. Therefore, the key factor for device performance is the depth and stability of $\mathrm{NV}^{-}$; successful detection depends on maintaining the dipole-dipole coupling strength by placing the $\mathrm{NV}^{-}$close to the surface while, at the same time, keeping the $\mathrm{NV}^{-}$stable.

We first calculate the depth dependence of $\mathrm{NV}^{-}$neutralization for a hydrogen terminated surface with only $\mathrm{NV}^{-}$and no nitrogen. This is done to isolate the $\mathrm{NV}^{-}$ neutralization process from nitrogen ionization process since both presences result in donating electrons.

The doping profile used here is a Gaussian distribution where the magnitude of the peak point and the standard deviation (straggle) are kept constant at each depth. The standard deviation is set to a much smaller value than the depth $(0.5 \mathrm{~nm})$. We use this doping profile to accurately determine the neutralization rate at each depth. In practice, such doping profile can be produced by chemical vapor deposition or a low energy ion implantation at shallow depths. The peak concentration is set at $10^{20}$ $\mathrm{cm}^{-3}$.

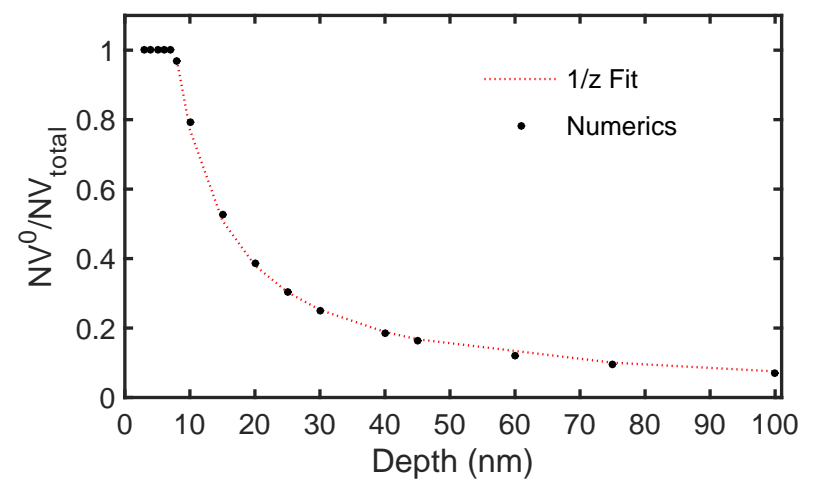

FIG. 3. Depth dependence of the $\mathrm{NV}^{-}$neutralization described by the neutralization ratio of $\mathrm{NV}^{-}$. The same Gaussian profile is used for the $\mathrm{NV}^{-}$distribution to evaluate neutralization at each depth. The standard deviation is $0.5 \mathrm{~nm}$ and the peak concentration is set at $10^{20} \mathrm{~cm}^{-3}$. Points represent values from the simulation; the dotted line represents the $1 / z$ fit. The neutralization ratio is taken as $\mathrm{NV}^{0} / \mathrm{NV}_{\text {total }}$, where $\mathrm{NV}^{0}$ is the concentration of $\mathrm{NV}^{0}$ and $\mathrm{NV}_{\text {total }}$ is the total concentration of NV centers.

Figure 3 shows the neutralization ratio, $\mathrm{NV}^{0} / \mathrm{NV}_{\text {total }}$, as a function of depth ranging from $3 \mathrm{~nm}$ to $100 \mathrm{~nm}$. There are two distinct regions: a plateau where all $\mathrm{NV}^{-} \mathrm{s}$ are completely neutralized to $\mathrm{NV}^{0} \mathrm{~s}$ and a monotonically decreasing region, where the neutralization is inversely proportional to the depth, $z$ (see Fig. 3). The $1 / z$ dependence indicates that the major driving force in neutralization is electrostatic.

The transition from the plateau to the monotonically decreasing region occurs when the potential difference generated by the accumulated holes becomes less than the total energy required to neutralize all $\mathrm{NV}^{-}$to $\mathrm{NV}^{0}$.

\section{Concentration Dependence}

As seen in Sec. IIIB, shallow depth promotes the neutralization of $\mathrm{NV}^{-}$. Since the amount of neutralization is governed by the electrostatic force, one way to offset neutralization is to increase concentration. Figure 4 shows the neutralization ratio as a function of $\mathrm{NV}^{-}$ concentration. The $\mathrm{NV}^{-}$profile is taken to be homogeneous throughout the diamond structure to remove any depth dependence. Concentrations of $\mathrm{NV}^{-}$ranging from $10^{16}$ to $10^{19} \mathrm{~cm}^{-3}$ are used to accommodate a recent experimental achievement of $50 \% \mathrm{NV}^{-}$conversion yield from doped nitrogen in a diamond sample ${ }^{39}$. The concentration of $10^{19} \mathrm{~cm}^{-3}$ is the maximum feasible doping concentration of nitrogen according to Element $\operatorname{Six}^{\mathrm{TM}}, 51$

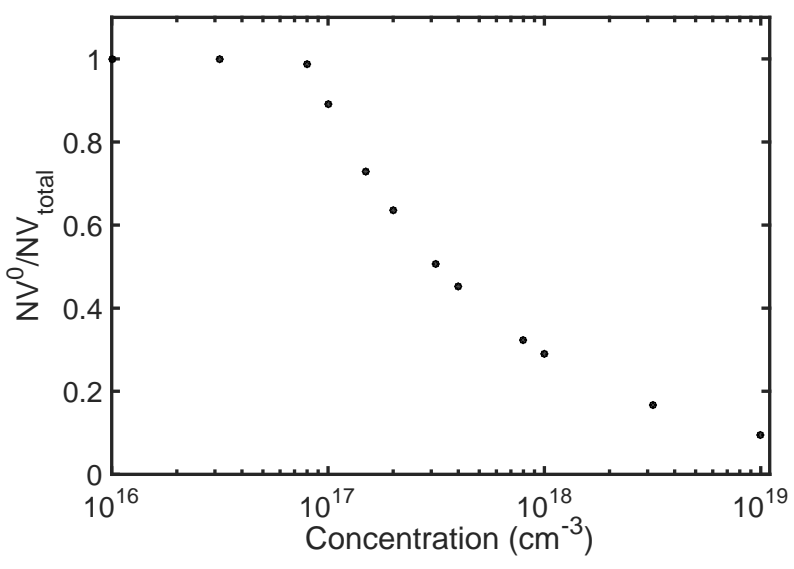

FIG. 4. Results of concentration dependence simulations. A homogeneous NV profile is used for each concentration. Concentration is plotted on a logarithmic scale. The neutralization ratio is shown as $\mathrm{NV}^{0} / \mathrm{NV}_{\text {total }}$, where $\mathrm{NV}_{\text {total }}$ is the total number of NV centers.

Figure 4 shows the concentration dependence of $\mathrm{NV}^{-}$ neutralization. As the concentration increases, the neutralization decreases. Increasing concentration decreases the depth of the fully neutralized region. Our calculation indicates that the total number of neutralized $\mathrm{NV}^{0}$ is less than the total number of accumulated hole created (the ratio of the number of the neutralized $\mathrm{NV}^{-} \mathrm{s}$ to the number of the accumulated holes is 0.76 to 1 ). We account this to the relatively high neutralization energy $\mathrm{NV}^{0}(2.67 \mathrm{eV})$. In Sec. IIIE, when we add nitrogen to the system, with ionization energy $1.7 \mathrm{eV}$, the ratio of the total number of the neutralized $\mathrm{NV}^{-}$plus the ionized nitrogen to the number of the accumulated holes is 1 to 1 .

\section{Effects of Concentration on Depth Dependence}

In Sec. IIIB, the overall concentration defined by the peak concentration and standard deviation of a Gaus- 
sian profile is kept the same at each depth. We now investigate how the peak concentration of $\mathrm{NV}^{-}$affects neutralization. We keep the same standard deviation of the Gaussian function as in Sec. IIIB while varying the peak concentration of the doping profile. We again assume that there is only $\mathrm{NV}^{-}$, with no nitrogen.

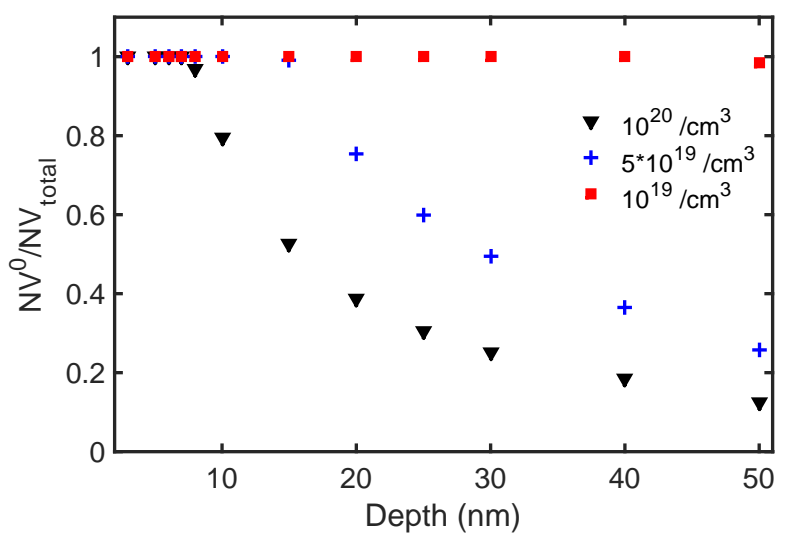

FIG. 5. Neutralization ratios of $\mathrm{NV}^{0} / \mathrm{NV}_{\text {total }}$ for each depth and peak concentration simulated. The diamond was modelled as being doped with only $\mathrm{NV}^{-}$. A Gaussian profile was used for the $\mathrm{NV}^{-}$distribution. The standard deviation was fixed at $0.5 \mathrm{~nm}$ for each profile, while varying the peak concentration.

Figure 5 shows the neutralization ratio as a function of depth at three slightly different concentrations. The figure indicates that neutralization occurs from the nearest surface preferentially as expected. Our results also suggest that slight changes in concentration produce drastic effects. With a five-fold increase in concentration, from $10^{19} \mathrm{~cm}^{-3}$ to $5 \times 10^{19} \mathrm{~cm}^{-3}$, the neutralization ratio changes from nearly complete neutralization to partial neutralization. Increasing the concentration from $10^{19}$ $\mathrm{cm}^{-3}$ to $10^{20} \mathrm{~cm}^{-3}$ pushes the neutralization curve further down reducing the neutralization ratio from 1 to 0.79 at a relatively shallow depth of $10 \mathrm{~nm}$. Therefore, one can control the stabilization of $\mathrm{NV}^{-}$at shallow depth by carefully controlling the peak concentration of $\mathrm{NV}^{-}$.

\section{E. Effects of Nitrogen}

Having modelled both the depth and concentration dependence of $\mathrm{NV}^{-}$neutralization, we now add nitrogen to the system. Again we use a homogeneous concentration profile for both nitrogen and $\mathrm{NV}^{-}$. The nitrogen concentration is fixed at $10^{18} \mathrm{~cm}^{-3}$. We examine neutralization with $0.1 \%, 1 \%$ and $10 \% \mathrm{NV}^{-}$yields from the nitrogen dopant.

Figure [6 shows only a $1 \%$ yield of $\mathrm{NV}^{-}$result, i.e., at a density of $10^{16} \mathrm{~cm}^{-3}$, for an easier view. Even though both $\mathrm{NV}^{-}$and nitrogen are homogeneously distributed

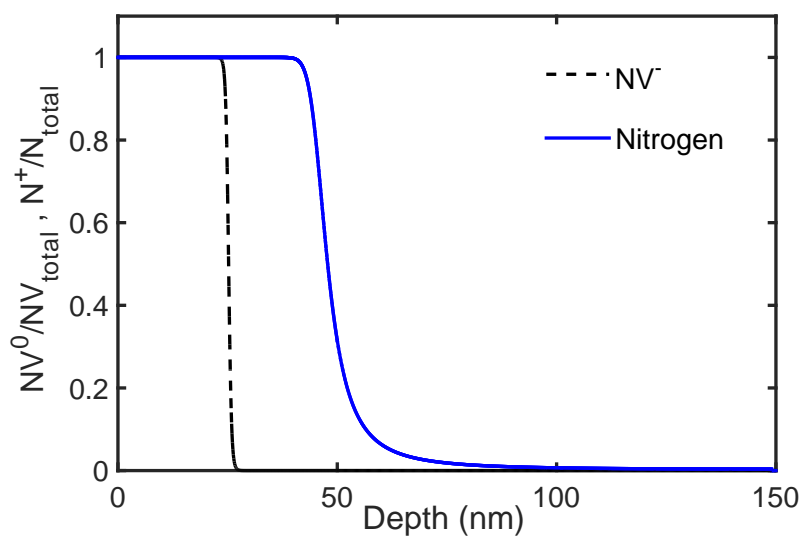

FIG. 6. Neutralization profile of nitrogen and $\mathrm{NV}^{-}$. The neutralization profile for NV centers is calculated as the ratio of $\mathrm{NV}^{0} / \mathrm{NV}_{\text {total }}$ at each depth, where $\mathrm{NV}^{0}$ is the total final concentration of $\mathrm{NV}^{0}$ and $\mathrm{NV}_{\text {total }}$ is the total initial concentration of NV centers. The neutralization profile of nitrogen is calculated as the ratio of $\mathrm{N}^{+} / \mathrm{N}_{\text {total }}$ at each depth, where $\mathrm{N}^{+}$is the total final concentration of $\mathrm{N}^{+}$and $\mathrm{N}_{\text {total }}$ is the total initial concentration of nitrogen.

throughout the diamond, one can see that nitrogen ionization is energetically favored over $\mathrm{NV}^{-}$neutralization. As briefly mentioned in Sec. IIC, the ionization energy of nitrogen is $1.7 \mathrm{eV}$ below the conduction band maximum (CBM), while the neutralization energy of $\mathrm{NV}^{-}$is $2.67 \mathrm{eV}$ below the CBM. Thus, the nitrogen will become ionized more easily than the $\mathrm{NV}^{-}$will be neutralized. In addition, because the ionization energy required for nitrogen is much larger compared to typical semiconductor dopants $(\ll 1 \mathrm{eV})$, the nitrogen dopant is hardly ionized at room temperature. As a result, there are enough nitrogen atoms left to ionize in preference to $\mathrm{NV}^{-}$by the hydrogen termination. Therefore, when nitrogen is introduced into a system with only $\mathrm{NV}^{-}$, the $\mathrm{NV}^{-}$neutralization is suppressed as long as the following conditions are both met: (a) the potential difference created by the electrostatic force, which depends on distance, cannot overcome the neutralization energy and (b) there are still some nitrogen atoms left for ionization.

Finally, by having a high nitrogen concentration, one can decrease neutralization at shallow depths. In principle, depths of less than $10 \mathrm{~nm}$ for stable $\mathrm{NV}^{-}$are viable.

\section{F. Effects of $\mathrm{pH}$}

In each of our models, we have taken the water layer to have a $\mathrm{pH}$ of 7 . In practice, the water layer is likely to take $\mathrm{pH}$ values near but not equal to $7: \mathrm{CO}_{2}$ from the air is dissolved in water can lower the $\mathrm{pH}$ of the water layer to 6 . Here, we examine $\mathrm{NV}^{-}$neutralization at a different $\mathrm{pH}$ (see Fig. 17). The band diagram is shown with only the valence bands and the Fermi energy level and around 


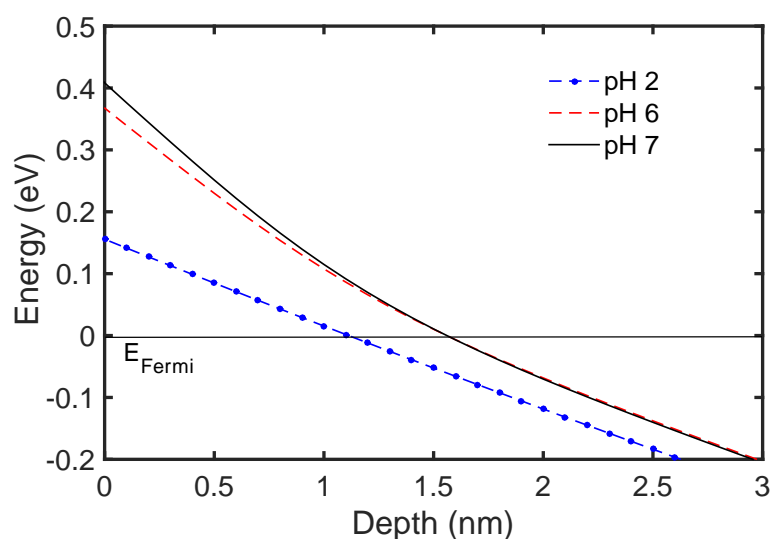

FIG. 7. Close-up of the band diagram around the 2-DHG region for diamond containing $\mathrm{NV}^{-}$at water $\mathrm{pH}=7$ (solid lines), $\mathrm{pH}=6$ (dashed lines) and $\mathrm{pH}=2$ (dotted lines), where the difference between the band diagrams can be seen.

the 2-DHG region (Fig. 7),

The band structures are very similar for the systems with the water layer at $\mathrm{pH} 6$ and $\mathrm{pH}$ 7. So, the concentrations of holes accumulated near the surface for both cases are also similar implying that neutralization of the $\mathrm{NV}^{-}$ would be similar. In fact, the neutralization ratio almost remains the same in the range of $\mathrm{pH} 6-8$. Therefore, neutralization of $\mathrm{NV}^{-}$is not affected by a $\mathrm{pH}$ change around $\mathrm{pH}$ 7. However, much lower $\mathrm{pH}$ has a large effect on band bending. In practice, much lower $\mathrm{pH}$ may be caused by a incomplete removal of a surface cleaning agent, such as sulfuric acid from the surface. For example, when the concentration $0.1 \mathrm{~mol} / \mathrm{L}$ of sulfuric acid is left on the surface, the $\mathrm{pH}$ of the water layer could drop down to as low as 2 . We see a change in the band bending and the drastic reduction of the 2 -DHG region at $\mathrm{pH}$ 2 (see Fig. 7).

\section{G. Conclusion}

We have investigated the neutralization mechanisms of $\mathrm{NV}^{-}$centers in diamond with a hydrogen terminated surface and a water layer on the surface. The hydrogen terminated diamond forms a 2 -DHG right below the interface. The depth of the 2-DHG changes with the dopant concentrations and distributions. The size of the 2-DHG reflects the amount of accumulated holes. A thin water layer condenses on the surface from the water vapor in the surrounding air.

The neutralization is quantified as the ratio of $\mathrm{NV}^{0}$ at equilibrium to the total number of $\mathrm{NV}$ originally implanted, $\mathrm{NV}^{0} / \mathrm{NV}_{\text {total }}$. The model is comprised of three regions, with appropriate equations used in each case: the Poisson equation for the bulk diamond region, the Schrödinger-Poisson equation for the hole accumulated region, and the Poisson-Boltzmann equation for the water layer.

We have calculated the neutralization ratio, $\mathrm{NV}^{0} / \mathrm{NV}_{\text {total }}$ at various depths. We find that the the $\mathrm{NV}^{-}$center neutralization is primarily electrostatic. For high initial concentrations of NV centers, we have found that $\mathrm{NV}^{-}$can remain stable even at depths of less than $10 \mathrm{~nm}$.

As the concentration increases, the neutralization decreases. The $\mathrm{NV}^{0}$ distribution as a function of depth shows that neutralization starts at sites near the surface as expected. Increasing concentration decreases the neutralization ratio. This is simply caused by the increase in the shear number of available $\mathrm{NV}^{-} \mathrm{S}$ as the concentration increases. However, when the depth dependence is examined with various but close concentrations, our results suggest that small changes in the initial concentration could lead to large changes in the $\mathrm{NV}^{-}$neutralization. The results are useful to control the stability of $\mathrm{NV}^{-}$at shallow depths.

The addition of nitrogen to the system shows that energetically, at a given depth, nitrogen ionization is favored over $\mathrm{NV}^{-}$neutralization due to the nitrogen ionization energy being lower than the $\mathrm{NV}^{-}$neutralization energy.

Finally, we have shown that small variations in the $\mathrm{pH}$ of the water layer will lead to insignificant changes in the 2-DHG region within the diamond. Such small changes mean that small variations in the $\mathrm{pH}$ of a water layer around $\mathrm{pH} 7$ for a hydrogen terminated diamond will not affect the neutralization of $\mathrm{NV}^{-}$centers within the diamond.

\section{ACKNOWLEDGMENTS}

This work is supported by NSF DMR-1505641. We are grateful to ITAMP at Harvard-Smithsonian Center for Astrophysics for hosting us during the summer, where the majority of this work has been carried out. We thank Hossein Sadeghpour, Mikhail Lukin and his group, and Ron Walsworth and his group for useful discussions and valuable input.

${ }^{1}$ G. Balasubramanian, P. Neumann, D. Twitchen, M. Markham, R. Kolesov, N. Mizuoschi, J. Isoya, J. Achard, J. Beck, J. Tissler, V. Jacques, P.R. Hemmer, F. Jelezko and J. Wrachtrup, Nature Materials 8, 383 (2009).

${ }^{2}$ C. L. Degen, Appl. Phys. Lett. 92, 243111 (2008).

${ }^{3}$ J. M. Taylor, P. Cappellaro, L. Childress, L. Jiang, D. Budker, P. R. Hemmer, A. Yacoby, R. Walsworth and M.D. Lukin, Nat. Phys. 7, 270, (2008).

${ }^{4}$ G. Balasubramanian, I. Y. Chan, R. Kolesov, M. Al-Hmoud, J. Tisler, C. Shin, C. Kim, A. Wojcik, P.R. Hemmer, A. Krueger, T. Hanke, A. Leitenstorfer, R. Bratschitsch, F. Jelezko and J. Wrachtrup, Nature 455, 648 (2008).

${ }^{5}$ J. R. Maze, P. L. Stanwix, J. S. Hodges, S. Hong, J. M. Taylor, P. Cappellaro, L. Jiang, M. V. Gurudev dutt, E. Togan, A. S. Zibrov, A. Yacoby, R. L. Walsworth and M. D. Lukin, Nature 455, 644 (2008).

${ }^{6}$ D. J. Maertz, A.P. Wijnheijmer, G. D. Fuchs, M. E. Nowakowski and D. D. Awschalom, Appl. Phys. Lett. 96, 092504 (2010). 
${ }^{7}$ C. A. Meriles, L. Jiang, G. Goldstein, J. S. Hodges, J. Maze, M. D. Lukin and P. Cappellaro, J. Chem. Phys. 133, 124105 (2010). ${ }^{8}$ S. Steinert, F. Dolde, P. Neumann, A. Aird, B. Naydenov, G. Balasubramanian, F. Jelezko and J. Wrachtrup, Rev. Sci. Instrum. 81, 043705 (2010).

${ }^{9}$ A. Laraoui, J. S. Hodges and C. A. Meriles, Appl. Phys. Lett. 97, 143104 (2010)

${ }^{10}$ L. T. Hall, C. D. Hill, J. H. Cole and L. C. L. Hollenberg, Phys. Rev. B 82, 045208 (2010).

${ }^{11}$ N. Zhao, J.-L. Hu, S.-W. Ho, J. T. K. Wan and R. B. Liu, Nat. Nanotechnol. 6, 242 (2011).

${ }^{12}$ L. P. McGuinness, Y. Yan, A. Stacey, D. A. Simpson, L. T. Hall, D. Maclaurin, S. Prawer, P. Mulvaney, J. Wrachtrup, F. Caruso, R.E. Scholten and L. C. L. Hollenberg, Nat. Nanotechnol. 6, 358 (2011).

${ }^{13}$ L. Ciobanu, D. A. Seeber and C. H. Pennington, J. Magn. Reson. 158, 178-182 (2002).

${ }^{14}$ Stephen J. DeVience, Linh M. Pham, Igor Lovchinsky, Alexander O. Sushkov and Nir Bar-Gill, Chinmay Belthangady, Francesco Casola, Madeleine Corbett, Huiliang Zhang, Mikhail Lukin, Hongkun Park, Amir Yacoby, and Ronald L. Walsworth, Nature Nanotechnology, 10, 129 (2015).

${ }^{15}$ M. Loretz, S. Pezzagna, J. Meijer and C. L. Degen, Nanoscale nuclear magnetic resonance with a 1.9-nm-deep nitrogen-vacancy sensor, Appl. Phys. Lett. 104, 033102 (2014).

${ }^{16}$ Viktor S. Perunicic, Liam T. Hall, David A. Simpson, Charles D. Hill and Lloyd C. L. Hollenberg, Phys. Rev. B 89, 054432 (2014).

${ }^{17}$ C. Müller, X. Kong, J.-M. Cai, K. Melentijević, A. Stacey, M. Markham, D. Twitchen, J. Isoya, S. Pezzagna, J. Meijer, J. F. Du, M. B. Plenio, B. Naydenov, L. P. McGuinness and F. Jelezko, Nat. Comm. 5, article 4703, (2014).

${ }^{18}$ Y. Mita, Phys. Rev. B 53, 11360 (1996).

${ }^{19}$ David M. Toyli, Christoph D. Weis, Gregory D. Fuchs, Thomas Schenkel and David D. Awschalom, Nano Lett. 10, 3168 (2010).

${ }^{20}$ D. Le Sage, K. Arai, D. R. Glenn, S. J. DeVience, L. M. Pham, L. Rahn-Lee, M. D. Lukin, A. Yacoby, A. Komeili and R. L. Walsworth, Nature 496, 486 (2013).

${ }^{21}$ P. Maletinsky, S. Hong, M. S. Grinolds, B. Hausmann, M. D. Lukin, R. L. Walsworth, M. Loncar and A. Yacoby, Nat. Nanotechnol. 7, 320 (2012).

${ }^{22}$ L. Rondin, J.-P. Tetienne1, P. Spinicelli, C. Dal Savio, K. Karrai, G. Dantelle, A. Thiaville, S. Rohart, J.-F. Roch and V. Jacques, Appl. Phys. Lett. 100, 153118 (2012)

${ }^{23}$ L. Rondin, J. P. Tetienne, S. Rohart, A. Thiaville, T. Hingant, P. Spinicelli, J.-F. Roch and V. Jacques, Nature Communications 4, 2279 (2013).

${ }^{24}$ M. S. Grinolds, S. Hong, P. Maletinsky, L. Luan, M. D. Lukin, R. L. Walsworth and A. Yacoby, Nat. Phys. 9, 215 (2013).

${ }^{25}$ K. Ohashi, T. Rosskopf, H. Watanabe, M. Loretz, Y. Tao, R. Hauert, S. Tomizawa, T. Ishikawa, J. Ishi-Hayase, S. Shikata, C. L. Degen, and K. M. Itoh, Nano Lett., 13 (10), 4733 (2013).

${ }^{26}$ Claire A. McLellan, Bryan A. Myers, Stephan Kraemer, Kenichi Ohno, David D. Awschalom, and Ania C. Bleszynski Jayich, Nano Lett., 16, 2450 (2016).
${ }^{27}$ B. Grotz, M. V. Hauf, M. Dankerl, B. Naydenov, S. Pezzagna, J. Meijer, F. Jelezko, J. Wrachtrup, M. Stutzmann, F. Reinhard, J. A. Garrido, Nat. Commun., 3, 729 (2012).

${ }^{28}$ M. V. Hauf, B. Grotz, B. Naydenov, M. Dankerl, S. Pezzagna, J. Meijer, F. Jelezko, J. Wrachtrup, M. Stutzmann, F. Reinhard, J. A. Garrido, Phys. Rev. B 83, 081304(R) (2011).

${ }^{29}$ K. M. C. Fu, C. Santori, P. E. Barclay, R. G. Beausoleil, Appl. Phys. Lett. 96, 121907 (2010).

${ }^{30}$ V. Petrakova, M. Nesladek, A. Taylor, F. Fendrych, P. Cigler, M. Ledvina, J. Vacik, J. Stursa, J. Kucka, Phys. Status Solidi A, 208, 2051 (2011)

${ }^{31}$ C. Bradac, T. Gaebel, C. I. Pakes, J. M. Say, A. V. Zvyagin, J. R. Rabeau, Small 9, 132 (2013).

${ }^{32}$ Moloud Kaviani, Peter Deák, Bálint Aradi, Thomas Frauenheim, Jyh-Pin Chou, and Adam Gali, Nano Lett. 14, 4772 (2014).

${ }^{33}$ L. Ciobanu, D. A. Seeber and C. H. Pennington, J. Magn. Reson. 158, 178-182 (2002).

${ }^{34}$ Stephen J. DeVience, Linh M. Pham, Igor Lovchinsky, Alexander O. Sushkov and Nir Bar-Gill, Chinmay Belthangady, Francesco Casola, Madeleine Corbett, Huiliang Zhang, Mikhail Lukin, Hongkun Park, Amir Yacoby, and Ronald L. Walsworth, Nature Nanotechnology 10, 129-134 (2015).

${ }^{35}$ M. Loretz, S. Pezzagna, J. Meijer and C. L. Degen, Appl. Phys. Lett. 104, 033102 (2014).

${ }^{36}$ A. R. Lang, M. Moore, A. P. W.Makepeace, W. Wierzchowski, and C. M. Welbourne, Phil. Trans. R. Soc. A. 337 (1648), 497 (1991).

${ }^{37}$ Konstantin Iakoubovskii and Guy J Adriaenssens, J. Phys.: Condens. Matter, 13 (26), 6015 (2001).

${ }^{38}$ A. M. Edmonds, U. F. S. D'Haenens-Johansson, R. J. Cruddace, M. E. Newton, K.-M. C. Fu, C. Santori, R. G. Beausoleil, D. J. Twitchen, and M. L. Markham, Phys. Rev. B 86, 035201 (2012).

${ }^{39}$ Claire A. McLellan, Bryan A. Myers, Stephan Kraemer, Kenichi Ohno, David D. Awschalom, and Ania C. Bleszynski Jayich, Nano Lett. 16 (4) 2450 (2016).

${ }^{40}$ F. Maier, J. Ristein, and L. Ley, Phys. Rev. B 64, 165411 (2001).

${ }^{41}$ J. Ristein, F. Maier, M. Riedel, J. B. Cui, and L. Ley, phys. stat. sol. (a) 181, 65 (2000).

${ }^{42}$ G. A. Baraff and Joel A. Appelbaum, Phys. Rev. B 5(2) 475 , (1971).

${ }^{43}$ C. E. Nebel, 1, B. Rezek, and A. Zrenner, Phys. Stat. Sol. (a) 201 (11), 2432-2438 (2004).

${ }^{44}$ G. Gouy, J. Phys. Radium, 9, 457, (1910).

${ }^{45}$ G. Gouy. Compt. Rend. 149, 654 (1910).

${ }^{46}$ David Leonard Chapman, Philos. Mag., 25 (148), 475 (1913).

${ }^{47}$ John David Jackson, Electrodynamics, 3rd. Ed. (John Wiley \& Sons, Inc, Hoboken, NJ, 1999).

${ }^{48}$ Andrew Zangwill, Modern electrodynamics, 1st. Ed. (Cambridge University Press, Cambridge, UK, 2012).

${ }^{49}$ Peter Deák, Bálint Aradi, Moloud Kaviani, Thomas Frauenheim, and Adam Gali, Phys. Rev. B 89, 075203 (2014)

${ }^{50} \mathrm{~S}$. Birner et al., IEEE Transactions on Electron Devices, 54 (9), 2137 (2007). also http://www.nextnano.com/

${ }^{51}$ The Element Six, CVD Diamond Handbook. online resorce http://www.e6cvd.com/ 